\title{
The first case of gastric carcinoma with NTRK rearrangement: identification of a novel ATP1B-NTRK1 fusion
}

\author{
Aya Shinozaki-Ushiku ${ }^{1} \cdot$ Shumpei Ishikawa ${ }^{2} \cdot$ Daisuke Komura $^{2} \cdot$ Yasuyuki Seto $^{3} \cdot$ Hiroyuki Aburatani $^{4}$. \\ Tetsuo Ushiku' ${ }^{1}$
}

Received: 12 February 2020 / Accepted: 9 March 2020 / Published online: 18 March 2020

(c) The International Gastric Cancer Association and The Japanese Gastric Cancer Association 2020

\begin{abstract}
NTRK gene rearrangements occur in a wide spectrum of tumors and are actionable events predictive of response to TRK inhibitor. We report the first case of gastric carcinoma harboring a NTRK fusion in a 79-year-old man. The tumor was composed predominantly of poorly cohesive carcinoma with focal tubular differentiation. Solid sheet-like or nested pattern of large oxyphilic cells was also noted in $10 \%$ of tumor. Pan-Trk immunohistochemistry demonstrated Trk expression with a diffuse cytoplasmic and dot-like staining only in the solid component. Extensive lymphatic invasion and multiple nodal metastases were noted and were predominated by Trk-positive component. A novel ATP1B1-NTRK1 fusion was detected by RNA-seq using fresh frozen sample. The patient died of the disease, despite surgery and chemotherapy. Although extremely rare, NTRK rearrangement does occur in gastric carcinoma and might be associated with aggressive phenotype as well as histologic features like solid growth with extensive lymphatic invasion.
\end{abstract}

Keywords Gastric carcinoma $\cdot$ NTRK1 $\cdot$ Fusion gene

\section{Introduction}

NTRK gene rearrangements have been reported in a broad spectrum of adult and pediatric tumors [1]. Although several earlier studies have investigated NTRK rearrangements in hundreds of gastric carcinomas, none of them detected fusion transcripts involving NTRK genes [2-4]. NTRK fusion causes constitutive activation of the TRK receptors and subsequent downstream pathways and plays an important role in oncogenesis. In addition, the presence of NTRK fusion has a great importance in a therapeutic aspect, since

Tetsuo Ushiku

usikut-tky@umin.ac.jp

1 Department of Pathology, Graduate School of Medicine, The University of Tokyo, 7-3-1 Hongo, Bunkyo-ku, Tokyo 113-0033, Japan

2 Department of Preventive Medicine, Graduate School of Medicine, The University of Tokyo, Tokyo, Japan

3 Department of Gastrointestinal Surgery, Graduate School of Medicine, The University of Tokyo, Tokyo, Japan

4 Genome Science Division, Research Center for Advanced Science and Technology, The University of Tokyo, Tokyo, Japan
TRK inhibitors, such as larotrectinib and entrectinib, are effective for these fusion-positive cancers with high responsive rate in a tumor agnostic fashion. Here, we present the first case of gastric carcinoma harboring NTRK rearrangement and describe the clinicopathologic features as well as genetic abnormalities including the detection of a novel ATP1B1-NTRK1 fusion by RNA sequencing.

\section{Case presentation}

A 79-year-old male presented with dysphagia and tarry stools lasting for 6 months. He underwent endoscopy to find a large ulcerated tumor in the cardia. Total gastrectomy with lymph node dissection was performed. Intraoperative cytology of ascites revealed negative for cancer and there was no metastatic lesion except positive lymph node metastases in the lesser curvature, along the proximal splenic artery and superior mesenteric vein. He was treated with adjuvant chemotherapy. 11 months after operation, he developed recurrent cancer in the left adrenal gland, peritoneal dissemination, and multiple metastases in the lymph nodes and bone. He died of the disease 13 months postoperatively. 


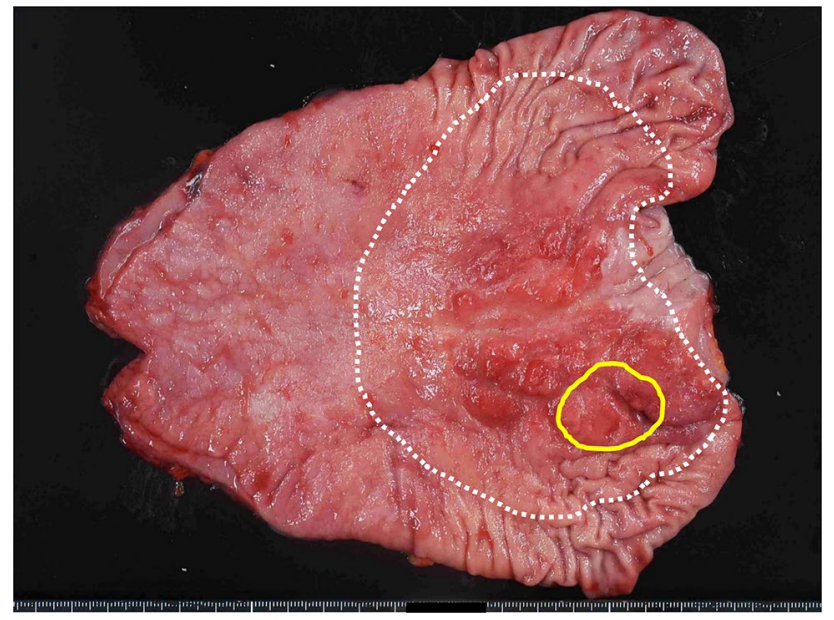

Fig. 1 Gross picture of gastrectomy specimen. The extent of the tumor spread is shown in dotted white line. Area marked with yellow line contained Trk-positive component

\section{Histologic findings}

Total gastrectomy specimen revealed a Borrmann type 3 tumor measuring $12 \times 10 \mathrm{~cm}$ in the proximal part of the stomach which invaded the esophagus and was exposed to the serosal surface (Fig. 1). Histologically, the tumor was predominantly composed of poorly cohesive adenocarcinoma which diffusely infiltrated the gastric wall. Focal gland-forming component was also noted in the superficial area of the tumor (Fig. 2a, b). Solid sheets or nests of cohesive cancer cells with abundant eosinophilic cytoplasm were also noted accounting for about $10 \%$ of the tumor, and this component exhibited extensive lymphatic invasion (Fig. 2c). Immunohistochemistry revealed that only the solid component was positive for pan-Trk, while poorly cohesive and glandular components were negative (Fig. 2d, e). The staining pattern was either weak/moderate diffuse cytoplasmic and/or strong dot-like in the cytoplasm, occasionally in a perinuclear fashion (Fig. 2f). No nuclear staining was found. Lymph node metastasis was present in 22 out of 47 nodes examined. Notably, the vast majority of the metastatic cancer cells involving the lymph nodes were diffusely pan-Trk-positive (Fig. 2g, h). Immunostaining of p53 was completely negative in all components (null pattern), suggesting TP53 gene mutation. E-cadherin expression was preserved and no HER2 staining was observed in all cancer cells. Expressions of mismatch repair proteins (MLH1, PMS2, MSH2, and MSH6) were retained and none of the neuroendocrine markers (chromogranin A, synaptophysin and CD56) were positive. EBER-ISH was negative. Background mucosa showed Helicobacter pylori-associated gastritis with intestinal
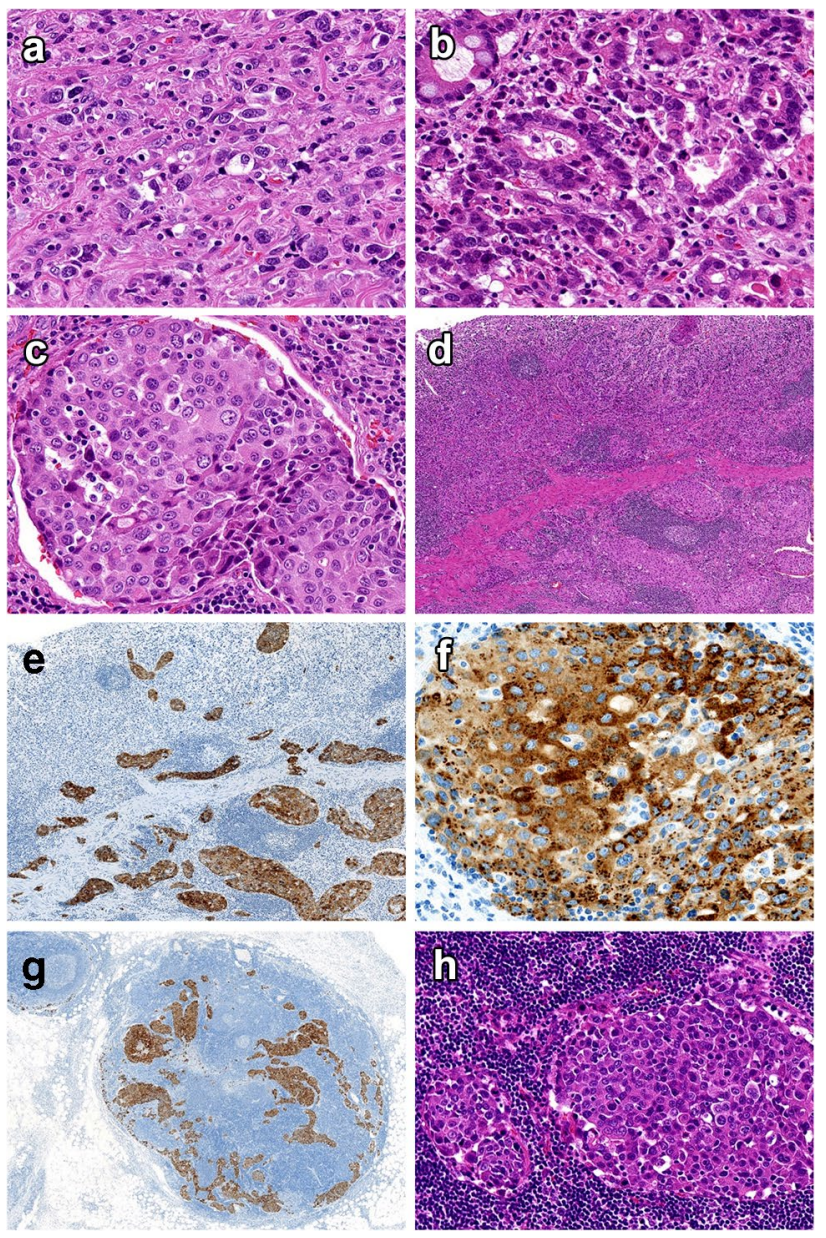

Fig. 2 Histologic pictures of the tumor. The predominant pattern was poorly cohesive carcinoma showing diffuse infiltration of cancer cells (a HE, $\times 400$ at original magnification). Intramucosal gland-forming component (b HE, $\times 400)$. Solid nests of cancer cells $(\mathbf{c} \mathrm{HE}, \times 400)$. Admixture of diffuse and solid components (d HE, $\times 100)$. Pan-Trk immunohistochemistry (IHC) revealed only solid component was positive for Trk $(\mathbf{e} \times 100)$. Cancer cells showing diffuse cytoplasmic and/or strong dot-like staining patterns $(\mathbf{f} \times 400)$. Trk-positive solid component predominated in the lymph node metastases (g Trk-IHC, scanning magnification, and h $\mathrm{HE}, \times 200$ )

metaplasia in the lower body and antrum. Immunohistochemistry for pan-Trk showed weak to moderate staining in the nerve plexus. Non-neoplastic gastric mucosa was negative for pan-Trk, while faint staining was observed in the fundic glands which is considered to be non-specific.

\section{Genetic findings}

RNA was extracted from frozen tumor tissue obtained from gastrectomy specimen and subjected to RNA-seq using Illumina GAIIx. Parabricks somatic pipeline version 2.4.3 and STAR-Fusion version 1.6.0 followed by Fusion Inspector were used for the discovery of somatic mutations 
and fusion transcripts, respectively. The raw sequencing reads were aligned to the reference human genome hg38. It revealed the presence of a novel gene fusion transcript, ATP1B1-NTRK1 (chr1:169,111,498-chr1:156,873,633 or chr1:169,111,498-chr1:156,874,571) (Fig. 3). These $A T P 1 B 1-N T R K 1$ fusion transcript consists of exons 1 and 2 of ATP1B1 gene and exons 8 or 10-17 of NTRK1 gene. The expected fusion protein contains transmembrane domain of ATP1B 1 and transmembrane domain and kinase domain of NTRK1 without protein frameshift. Other mutations identified by RNA-seq were as follows; ARIDIA (c.3142delC, p.P1048fs), SNRNP200 (c.4448G > A, p.R1483H), ARRDC1 (c.1169G > A, p.R390H), and A2M (c.3351_3352insT, p.R1118fs). Immunohistochemistry revealed loss of ARID1A expression in a focal tumor area (about $10 \%$ of pan-Trk-negative component), suggesting that this mutation may also play a certain role in the oncogenesis or tumor progression. None of other three mutations are reported to be pathogenic mutations.

\section{Discussion}

Specific type of NTRK fusions occurs in several rare tumors at high frequencies, e.g., ETV6-NTRK3 fusion in secretary carcinomas of the breast and salivary gland, infantile fibrosarcomas, and congenital mesoblastic nephromas. In addition, NTRK rearrangements are also found in various common cancers in very low frequencies (e.g., lung cancer, colon cancer, breast cancer, and melanoma in less than 1\%) [1-3]. Several studies have analyzed large series of gastric cancer for NTRK fusions but failed to detect fusion transcripts [2-4]. For example, none of 414 gastric cancers from The Cancer Genome Atlas database harbors NTRK fusion gene [2]. Lee et al. reported that one out of 66 gastric carcinomas showed positive TrkA immunostaining and demonstrated break apart of NTRK1 gene by fluorescence in situ hybridization; however, the fusion transcript was not investigated [5]. The present case is the first to demonstrate NTRK fusion in gastric carcinoma and is also the first to identify novel fusion, ATP1B1-NTRK1.

$A T P 1 B 1$ gene encodes the beta subunit of $\mathrm{Na}^{+} / \mathrm{K}^{+}$-ATPase and is ubiquitously expressed. This gene is known to be a fusion partner of PRKACA and PRKACAB in oncocytic papillary neoplasms of the pancreas and bile duct, and a partner of NRG1 in cholangiocarcinoma; however, fusion with $N T R K 1$ has not been reported [6, 7]. Immunohistochemistry with pan-Trk antibody is useful to screen NTRK fusions and most fusion-positive tumors show cytoplasmic staining. In addition, characteristic staining patterns have been reported, i.e., nuclear staining in ETV6-NTRK3 fusion, nuclear membrane staining in $L M N A-N T R K 1$ fusion, and membranous staining in TPM3/4-NTRK1/3 fusion [8]. The dot-like staining pattern observed in the present case has not been reported previously, and this staining pattern may characterize this novel ATP1B1-NTRK1 fusion.
Fig. 3 Schematic presentation of $A T B 1 B-N T R K 1$ fusion transcript and predicted fusion protein

\section{ATP1B1-NTRK1 Fusion Transcript}

Based on ENST00000367815.9(ATP1B1) ENST00000524377.5(NTRK1)

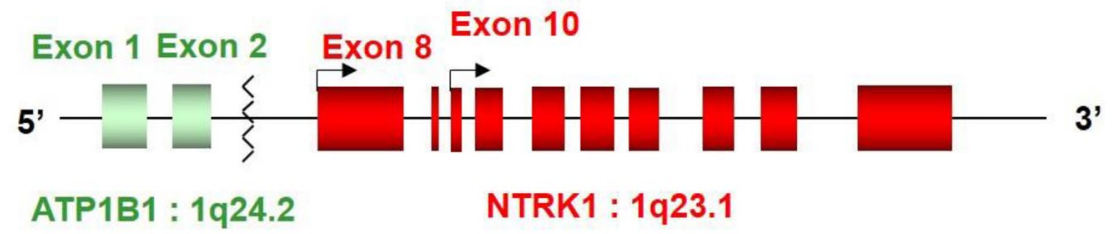

ATP1B1-NTRK1 Fusion Protein (Prediction)

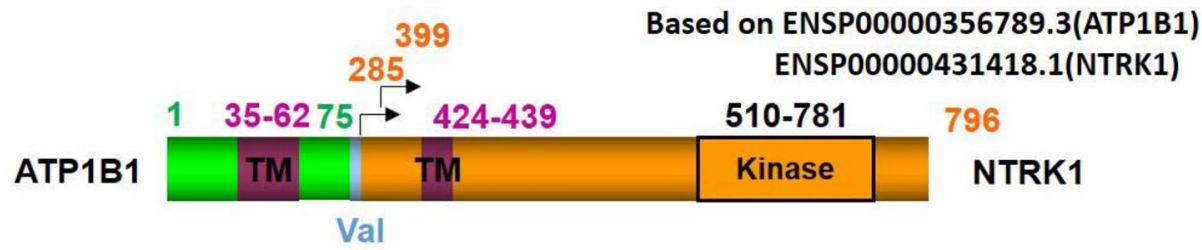


Another interesting finding was heterogeneous Trk expression in the tumor with only solid component stained positively, while all components showed p53 null pattern. Based on this, only solid component is highly likely to have gained ATP1B1-NTRK1 fusion during the tumor development. Although mutation of TP53 was not detected by RNA sequencing, completely negative staining of p53 suggested TP53 gene alterations such as frameshift, non-sense mutation, and LOH [9-11]. Furthermore, the Trk-positive component showed extensive lymphatic invasion and metastasis to the lymph nodes. These observations suggest that TP53 alteration occurred at early in the oncogenesis, and subsequent development of a subclone with NTRK1 fusion gained more aggressive phenotype in this case.

The presence of NTRK fusion is crucial in selecting the treatment options since TRK inhibitors achieve high response rates. Although there are a few studies with detailed clinicopathologic evaluation of NTRK fusion-positive carcinomas other than secretary carcinoma of salivary gland, some features are described in common: poorly differentiated, high-grade histology, solid growth pattern, frequent lymphovascular invasion and lymph node metastasis, and poor prognosis, in line with the present case [12-16]. Although these findings should be validated in a larger series, these features in pathological examination might facilitate performing pan-Trk immunohistochemistry to select tumors appropriate for further NGS assay, whereas the rarity raises a challenge for a routine screening approach.

In conclusion, although exceptionally rare, $N T R K$ fusion can occur in gastric carcinoma and identifying this fusion will benefit the patient outcome [17, 18]. Poorly differentiated, solid growth pattern with extensive lymphatic invasion may be histologic features for suspicion of NTRK fusion and for facilitating screening with pan-Trk immunohistochemistry.

Funding This study was supported by AMED Project for Cancer Research and Therapeutic Evolution (P-CREATE) $(19 \mathrm{~cm} 0106531 \mathrm{~h} 0001)$, and JSPS KAKENHI Grant-in-Aid for Scientific Research (A) 16H02481 (SI).

\section{Compliance with ethical standards}

Conflict of interest The authors declare that they have no conflict of interest.

Ethical approval This study was conducted with the approval of the local ethics committee of The University of Tokyo in accordance with the Helsinki Declaration.

\section{References}

1. Cocco E, Scaltriti M, Drilon A. NTRK fusion-positive cancers and TRK inhibitor therapy. Nat Rev Clin Oncol. 2018;15:731-47.

2. Okamura R, Boichard A, Kato S, Sicklick JK, Bazhenova L, Kurzrock R. Analysis of NTRK alterations in pan-cancer adult and pediatric malignancies: implications for NTRK-targeted therapeutics. JCO Precis Oncol. 2018. https://doi.org/10.1200/PO.18.00183.

3. Solomon JP, Linkov I, Rosado A, Mullaney K, Rosen EY, Frosina D, et al. NTRK fusion detection across multiple assays and 33,997 cases: diagnostic implications and pitfalls. Mod Pathol. 2020;33:38-46.

4. Zehir A, Benayed R, Shah RH, Syed A, Middha S, Kim HR, et al. Mutational landscape of metastatic cancer revealed from prospective clinical sequencing of 10,000 patients. Nat Med. 2017;23:703-13.

5. Lee SJ, Li GG, Kim ST, Hong ME, Jang J, Yoon N, et al. NTRK1 rearrangement in colorectal cancer patients: evidence for actionable target using patient-derived tumor cell line. Oncotarget. 2015;6:39028-35.

6. Singhi AD, Wood LD, Parks E, Torbenson MS, Felsenstein M, Hruban RH, et al. Recurrent rearrangements in PRKACA and PRKACB in intraductal oncocytic papillary neoplasms of the pancreas and bile duct. Gastroenterology. 2020;158:573-82.

7. Vyas M, Hechtman JF, Zhang Y, Benayed R, Yavas A, Askan G, et al. DNAJB1-PRKACA fusions occur in oncocytic pancreatic and biliary neoplasms and are not specific for fibrolamellar hepatocellular carcinoma. Mod Pathol. 2019. https://doi.org/10.1038/s4137 9-019-0398-2.

8. Hechtman JF, Benayed R, Hyman DM, Drilon A, Zehir A, Frosina $\mathrm{D}$, et al. Pan-Trk immunohistochemistry is an efficient and reliable screen for the detection of NTRK fusions. Am J Surg Pathol. 2017;41:1547-51.

9. Murnyak B, Hortobagyi T. Immunohistochemical correlates of TP53 somatic mutations in cancer. Oncotarget. 2016;7:64910-20.

10. Lopez I, Oliveira LP, Tucci P, Alvarez-Valin F, Coudry RA, Marin M. Different mutation profiles associated to P53 accumulation in colorectal cancer. Gene. 2012;499:81-7.

11. Nakamura M, Obata T, Daikoku T, Fujiwara $H$. The association and significance of p53 in gynecologic cancers: the potential of targeted therapy. Int J Mol Sci. 2019;20:5482.

12. Pietrantonio F, Di Nicolantonio F, Schrock AB, Lee J, Tejpar S, Sartore-Bianchi A, et al. ALK, ROS1, and NTRK rearrangements in metastatic colorectal cancer. J Nat Cancer Inst. 2017;109:1-10.

13. Farago AF, Taylor MS, Doebele RC, Zhu VW, Kummar S, Spira $\mathrm{AI}$, et al. Clinicopathologic features of non-small-cell lung cancer harboring an NTRK gene fusion. JCO Prec Oncol. 2018. https://doi. org/10.1200/PO.18.00037.

14. Chou A, Fraser T, Ahadi M, Fuchs T, Sioson L, Clarkson A, et al. NTRK gene rearrangements are highly enriched in MLH1/ PMS2 deficient, $B R A F$ wild-type colorectal carcinomas-a study of 4569 cases. Mod Pathol. 2019. https://doi.org/10.1038/s4137 9-019-0417-3.

15. Lasota J, Chlopek M, Lamoureux J, Christiansen J, Kowalik A, Wasag B, et al. Colonic adenocarcinomas harboring NTRK fusion genes: a clinicopathologic and molecular genetic study of 16 cases and review of the literature. Am J Surg Pathol. 2020;44:162-73.

16. Yeh YA, Yang S, Constantinescu M, Chaudoir C, Tanner A, Henry $\mathrm{M}$, et al. Prostatic adenocarcinoma with novel NTRK3 gene fusion: a case report. Am J Clin Exp Urol. 2019;7:341-5.

17. Okugawa Y, Tanaka K, Inoue Y, Kawamura M, Kawamoto A, Hiro J, et al. Brain-derived neurotrophic factor/tropomyosin-related kinase B pathway in gastric cancer. Br J Cancer. 2013;108:121-30.

18. Hayakawa Y, Sakitani K, Konishi M, Asfaha S, Niikura R, Tomita $\mathrm{H}$, et al. Nerve growth factor promotes gastric tumorigenesis through aberrant cholinergic signaling. Cancer Cell. 2017;31:21-34.

Publisher's Note Springer Nature remains neutral with regard to jurisdictional claims in published maps and institutional affiliations. 